Meta

Journal des traducteurs

Translators' Journal

\title{
Quand la psychanalyse entre dans la traduction
}

\section{Pier-Pascale Boulanger}

Volume 54, numéro 4, décembre 2009

URI : https://id.erudit.org/iderudit/038901ar

DOI : https://doi.org/10.7202/038901ar

Aller au sommaire du numéro

\section{Éditeur(s)}

Les Presses de l'Université de Montréal

ISSN

0026-0452 (imprimé)

1492-1421 (numérique)

Découvrir la revue

Citer cet article

Boulanger, P.-P. (2009). Quand la psychanalyse entre dans la traduction. Meta, 54(4), 733-752. https://doi.org/10.7202/038901ar

\section{Résumé de l'article}

L'être humain est produit par le langage davantage qu'il ne le produit. Lorsque ce constat psychanalytique est posé sur le terrain de la traductologie, celle-ci est amenée à envisager des sujets habituellement considérés comme étant hors de son champ de compétence, tels que les lapsus. Dans le sillage des travaux de François Peraldi, nous montrons les affinités épistémologiques et conceptuelles de la psychanalyse et de la traduction et, plus encore, la nécessité pour la traduction d'incorporer certains vecteurs de réflexion psychanalytiques. Dans cette visée, nous relançons l'injonction que Peraldi a faite aux traducteurs et qui rejoint la poétique du traduire d'Henri Meschonnic : se mettre à l'écoute du corps de la parole et de la parole du corps dans le langage. Cette écoute doit passer par une sensibilisation du corps traduisant, par l'éveil de sa fonction érotique, qui se trouve à l'état latent. 


\title{
Quand la psychanalyse entre dans la traduction
}

\author{
PIER-PASCALE BOULANGER \\ Université Concordia, Montréal, Canada \\ pierpascale.boulanger@concordia.ca
}

\begin{abstract}
RÉSUMÉ
L'être humain est produit par le langage davantage qu'il ne le produit. Lorsque ce constat psychanalytique est posé sur le terrain de la traductologie, celle-ci est amenée à envisager des sujets habituellement considérés comme étant hors de son champ de compétence, tels que les lapsus. Dans le sillage des travaux de François Peraldi, nous montrons les affinités épistémologiques et conceptuelles de la psychanalyse et de la traduction et, plus encore, la nécessité pour la traduction d'incorporer certains vecteurs de réflexion psychanalytiques. Dans cette visée, nous relançons l'injonction que Peraldi a faite aux traducteurs et qui rejoint la poétique du traduire d'Henri Meschonnic: se mettre à l'écoute du corps de la parole et de la parole du corps dans le langage. Cette écoute doit passer par une sensibilisation du corps traduisant, par l'éveil de sa fonction érotique, qui se trouve à l'état latent.
\end{abstract}

\section{ABSTRACT}

Humans are produced by language rather than produce it. When this psychoanalytical contention is brought to translation theory, it pushes the latter to consider objects usually deemed out of its conceptual reach, such as slips of the pen. In the wake of François Peraldi, we stress the epistemological and conceptual affinities that psychoanalysis and translation share, but more so the necessity for translation to integrate certain psychoanalytical viewpoints. Like Peraldi we urge translators to give ear to the body of the word and the word of the body, a stance which also builds on Henri Meschonnic's poetics of translation. The ability to listen is only possible if the body translating becomes sensitized, if its erotic function is aroused from its latent state.

\section{MOTS-CLÉS/KEYWORDS}

corporalité du langage, langage du corps, écoute du signifiant, érotique du traduire, lapsus translatandi

corporality of language, body language, listening to the signifier, erotics of translating, lapsus translatandi

Il faut des années pour se démettre de l'écoute du sens des langages, de leur fonction d'information, de communication, et pour se laisser toucher. Après tout, le son est matériel au même titre que le caillou puisque comme lui il peut briser un verre. Il faut se laisser toucher dans son corps et, plus précisément dans son corps érotique, par les mots-corps, les mots-sexes des autres et de ces autres dont c'est l'unique langue, je veux parler des psychotiques.

(Peraldi 1978a: 13')

Ainsi parlait Peraldi, François de son prénom. Que le son soit matériel, donc que l'on puisse par lui être touché, Peraldi devait le confirmer chaque fois qu'il prenait la parole, qu'il avait par ailleurs fort mélodieuse (Jbeili 2005: 96). Lorsque Peraldi oppose l'écoute du sens des langages à la capacité de se laisser toucher, il critique l'attachement de l'auditeur au seul contenu lexical des mots, qu'il dénonce comme 
l'assujettissement au pouvoir symbolique de la fonction d'échange du langage. Hors de toute visée communicationnelle du langage assurant la transmission d'un sens grâce à sa fonction de représentation, le psychotique utilise les mots «comme des choses, bonnes ou mauvaises, à dévorer ou à expulser»(Grenier 2002: 138). Que demande le fou? Qu'à être entendu. En réponse à cet "appel à l'écoute» (Peraldi 1978a: 6), tout autre qu'un appel à l'aide, le psychanalyste doit être disposé à recevoir et à percevoir l'effet de certains signifiants sur son corps. Ainsi, Peraldi écoutant pour la énième fois la colère du jeune schizophrène Norbert contre les sept maîtres $d u$ monde qui le tenaient sous leur domination s'est senti mordu par les dentales et les sifflantes des "mots-morsures" proférés par ce jeune homme assis devant lui raide sur sa chaise, les dents découvertes, sifflant de rage comme un serpent (Peraldi 1978a: 10-11). Eût-il cherché à donner un sens au signifié récurrent «les sept maîtres du monde», l'analyste n'aurait pas senti les effets de la parole de Norbert, et l'analyse n'aurait pas connu le cours favorable qui a suivi. De fait, Peraldi relate la réaction surprenante de Norbert, qui, en l'entendant dire qu'il était en train de le mordre, moment cathartique, s'est soudainement tu pour ensuite parler de sa mère qu'enfant il mordait.

Contrer l'habitude de chercher le référent des mots signifie pour le psychanalyste qu'il s'affranchisse de l'emprise de la fonction d'échange du langage en prenant conscience du conditionnement acquis au signifié. Cela implique aussi la reconnaissance d'autres modes de captation de sens, telle l'écoute des signifiants, c'est-à-dire la sensibilité à la matérialité sonore donc physique du langage. Cette sensibilité auditive, Peraldi la place au cœur même de la pratique de l'analyste et du traducteur en tant qu'ils travaillent tous deux dans le langage et sont travaillés par lui. Il nommera l'érotique «la tentative de dégager la logique des investissements pulsionnels du signifiant et de leur ordonnancement dans le progrès syntagmatique» (Peraldi 1982a: 24).

Habile et novateur, Peraldi crée un lieu de rencontre entre la psychanalyse et la théorie de la traduction, qui dans les années 1980 n'avait toujours pas reçu l'appellation traductologie et n'évoluait pas encore dans l'interdisciplinarité qu'on lui connaît aujourd'hui. Linguiste formé sous la direction de Barthes et psychanalyste émoulu de l'École freudienne de Paris, Peraldi mène de front une pratique privée et une carrière de professeur à l'École de traduction de l'Université de Montréal de la fin des années 1970 jusqu'en 1991, deux ans avant sa mort. Actif dans le milieu intellectuel montréalais, il met sur pied le réseau des cartels suivant le modèle proposé par Lacan et par cette voie il organise, de 1981 à 1988, un séminaire sur le sujet l'Autre, le temps et la mort. Y sont invités des apprentis analystes et des psychanalystes appartenant à la marge analytique montréalaise, c'est-à-dire ceux qui ont choisi de ne pas adhérer à une institution reconnue (Ducharme 2005: 26), en l'occurrence la Société canadienne de psychanalyse, branche de l'Association internationale de psychanalyse. En 1982, Peraldi dirige un numéro de Meta dans le but de conjoindre ces deux disciplines autour de leur dénominateur commun: la fonction de la parole dans le champ du langage. Dans le parcours de sa pensée, Peraldi écrit profusément dans des périodiques d'horizons divers, portant sa réflexion sur un nombre considérable de thèmes et questions ${ }^{2}$, et il s'impose comme le premier à avoir théorisé la traduction par les moyens de la psychanalyse. Michaud lui a rendu hommage en 1998, dirigeant un numéro de TTR autour du thème des voies de traverse reliant traduction et psychanalyse. Depuis cette décennie, c'est le silence théorique, et les perspectives psychanalytiques dépassant le 
cadre restreint de la traduction de l'œuvre freudienne demeurent fragmentaires, parsemant çà et là des monographies et des articles ${ }^{3}$ sans jamais se consolider en paradigme parmi ceux qui parcourent la traductologie.

Reconnaissons pourtant que pour peu que l'on s'intéresse à la dynamique subjective du traduire, certains phénomènes psychiques relevés par Freud et touchant le quotidien du traducteur dans sa pratique ne manqueront pas d'interpeller les traductologues. Au nombre des actes manqués et des lapsus qui sont «constitutifs du clivage irrémédiable du sujet entre l'inconscient [...] et le moi» (Peraldi 1982a: 3), et certainement actifs dans la praxis du sujet traduisant, figurent les omissions, les faux sens, les contresens et les non-sens, les barbarismes et l'oubli momentané du mot juste. L'origine des sentiments d'humilité, de culpabilité et d'angoisse face à la tâche du traducteur ainsi que le mécanisme de refoulement par l'autocensure se présentent comme autant de questions auxquelles la traductologie se trouve mal outillée pour répondre et que d'aucuns excluront vite de son champ de compétence. Quant au désir animant l'activité traduisante et qu'en 1977, Gavronsky plaçait radicalement du côté de la piété et de la soumission à l'autorité paternelle du texte ou du cannibalisme et de la transgression de celle-ci, les traductologues l'ont peu exploré jusqu'à présent. Les traducteurs s'alimentent-ils tous du même supradésir: se faire l'auteur original d'un texte étranger, comme l'avance partiellement Venuti (2002)? Le cadre conceptuel dans lequel poser ces questions et l'outillage théorique pour les faire travailler sont à chercher du côté de la psychanalyse.

Si le titre du présent article annonce le dépassement de la simple conjonction de la psychanalyse et de la traduction, c'est qu'il ne suffit plus de conjoindre les deux disciplines: il faut introduire l'une dans l'autre. Ainsi, nous nous attacherons dans un premier temps à montrer les affinités épistémologiques, théoriques et politiques pour ensuite passer par la psychanalyse afin de mettre en lumière des phénomènes généralement tenus à l'écart, tels que les échappées de parole singulière (Fenoglio 2001: 178) et le refoulement par l'otocensure. Dans cette visée seront présentés les enjeux que défendait Peraldi quant au statut épistémologique de la psychanalyse au regard du savoir médical, rapport de force dans lequel la traductologie reconnaîtra le reflet de son rapport à la science linguistique. Dans un deuxième temps, nous développerons le syllogisme selon lequel traduire engage le sujet dans une pratique langagière double: lire en vue d'écrire et écrire en vue d'être lu. Or, depuis qu'il a découvert l'inconscient, Freud a postulé que l'être humain n'est pas maître de sa parole, c'est-à-dire qu'il ne produit pas le langage, mais qu'il est produit par lui, et ce malgré ses difficultés à l'admettre en tant que personne sociale autonome ayant conscience de son unité (Freud 1922/1961: 8). Donc, le sujet traduisant ne maîtrise ni la lecture du texte original ni l'écriture de sa traduction. Comment alors cette non-maîtrise se joue-t-elle? Nous proposons de chercher des éléments de réponse dans les lapsus translatandi.

Tout autant que le psychanalyste, le traducteur doit pouvoir lire et entendre autrement: être sensible au signifiant, donc au corps de la parole, comme il a été cité en exergue. Peraldi en appelait au corps érotique potentiel chez tout sujet, seul capable par l'écoute et le silence de se laisser toucher. Peut-on amener le sujet traduisant à découvrir ce corps dans la visée de ce qu'on appellera une érotique du traduire? Cela signifierait sensibiliser, érotiser le corps afin d'en potentialiser les capteurs de sens, en commençant par l'oreille que nous avons atrophiée. Quant à l'écriture, 
toujours suivant l'axiome freudien, comment le sujet est-il écrit plutôt qu'il n'écrit? Cette question nous amènera à envisager le corps de la parole dans le langage et la parole du corps dans le langage.

\section{Psychanalyse et traduction}

Hormis le fait que Freud n'a jamais consacré à la traduction une réflexion particulière sur son mode de fonctionnement, il la concevait comme étant au cœur des processus psychiques et de l'activité analytique. Sans avoir défini précisément ce qu'il entendait par traduction, ni Lacan après lui d'ailleurs, Freud englobe dans l'Übersetzung les mécanismes de l'appareil psychique qui transforment les idées et les affects en mots ou encore transposent les contenus psychiques du registre inconscient au registre conscient.

Radicalement liées, la traduction et la psychanalyse mettent en jeu la matière langagière dans sa possibilité de transformation, et c'est dans l'attention particulière portée à cette dynamique que leur corrélation se trouve légitimée. Selon le premier modèle de l'appareil psychique chez Freud, l'inconscient conserve ou laisse passer à la conscience toute pensée, image sonore ou matérielle de mots, ou tout souvenir (trace mnésique) selon qu'ils rencontrent une résistance particulière lorsqu'ils présentent une menace de déplaisir. Le blocage dans l'inconscient des matériaux psychiques à risque qui tentent de s'actualiser dans la conscience résulte d'un processus défensif: le refoulement. Contrairement à l'idée dépréciative de l'inconscient comme poubelle, l'activité de celui-ci dépasse largement la fonction de récipient passif, du fait qu'il déplace et condense les matériaux psychiques, les organisant ainsi très librement. Il les archive sans ordre chronologique ni contradiction logique et les conserve à l'abri du passage du temps. En cela il est dit avoir sa propre syntaxe, comme un langage ses propres règles d'agencement.

Insolubles, les matériaux refoulés exercent une pression, de sorte qu'ils finissent par s'exprimer sous le mode particulier du symptôme psychonévrotique, de l'acte manqué, du rêve, du lapsus et du Witz ou mot d'esprit (auquel Lacan préférera trait d'esprit). Freud considère ces trois derniers modes d'expression comme autant de voies de traduction des contenus inconscients qui marquent le passage du langage somatique au langage verbal et se prêtent à l'interprétation du psychanalyste. Au fil de sa pratique clinique, Freud constatera que le contenu de la parole - ce qu'on attribue traditionnellement au signifié - est flou, alors qu'au contraire l'image matérielle des mots, leur forme visuelle et sonore, est rigoureusement structurée, donc lisible et analysable. Ce constat révolutionnaire, Freud le fera envers et contre toute la métaphysique traditionnelle fondée sur ce que Jacques Derrida condensera dans le vocable logocentrisme. À l'instar du psychanalyste, le traducteur se met à l'écoute de cette parole, demeurant sensible à la matière orale $d u$ sens (Meschonnic 1989: 49), à sa matérialité, dont certains signifiants capteront son attention et, partant, qu'il s'essaiera à rendre. Mais attention: cette méthodologie diffère nettement d'une herméneutique du caché attachée à construire un sens symbolique sur la base du fait que l'auteur avait voulu dire autre chose que ce qui se révèle à la lecture (Peraldi 1982a: 126). Lorsque la lecture psychanalytique du texte littéraire s'engage sur la pente glissante des interprétations symboliques réductrices, elle devient science des profondeurs, épithète désobligeante que la psychanalyse aurait méritée plus souvent qu'à son tour. 
En clair, la grande découverte de Freud a été d'avancer que le sujet n'est pas maître de sa parole et qu'il se trouve largement sous l'emprise de l'inconscient. $A$ contrario, l'être humain en revient toujours à se croire capable de maîtriser ses dires. À propos de cette illusion persistante, Lavie, en préface à la traduction française du livre Le mot d'esprit de Freud écrit ceci: «Pas plus que celui de 1905, le lecteur d'aujourd'hui ne peut accepter que sa parole ne soit pas exactement le reflet de ce qu'il veut lui faire dire, ni qu'elle exprime autre chose, et encore moins que l'autre chose lui échappe» (Lavie 1905/1988: 19). Au risque d'enfoncer une porte ouverte, rappelons qu'à l'affirmation de Freud les tenants de la théorie interprétative opposeraient une fin de non-recevoir, invoquant à l'instar de Seleskovitch et Lederer (1984: 18) que " [s]i l'on parle c'est avec l'intention de communiquer; il n'est pas de message qui ne vise à transmettre un sens». Cela revient donc à dire que le sens de la parole de l'auteur est l'objet du traducteur, qu'il doit réexprimer afin d'établir la communication, de faire passer le message. Traditionnellement, cette tâche interprétative s'appuie sur la présupposition que l'auteur savait ce qu'il voulait dire. Dans l'intention de communiquer, il a sciemment proportionné l'explicite de sa formulation par rapport au non-dit (Seleskovitch et Lederer 1984: 22), levant ainsi la possibilité de polysémie interprétative, celle-là même qui gêne tant la tâche du traducteur. Avec des lunettes freudiennes, on peut relire, mais à la lettre, l'affirmation selon laquelle «en général le vouloir dire n'est pas équivoque et cherche à se manifester, à se faire comprendre» (Seleskovitch et Lederer 1984: 24) et signaler l'animisme qui confère soudainement au vouloir dire le statut de sujet. Ils seraient donc deux à parler: l'auteur et son vouloir dire. C'est précisément cet excès de parole qui intéresse le psychanalyste et auquel Peraldi travaillait à intéresser la traduction. L'École du sens a soutenu une théorie communicationnelle aujourd'hui largement admise et enseignée fondée sur la méfiance de la structure formelle de la langue, puisque la fidélité au mot se pose comme le grand obstacle pour la traduction et une source d'interférences (Seleskovitch et Lederer 1984: 32).

Neutraliser les interférences: n'est-il pas tout à fait louable de s'y appliquer surtout lorsqu'elles menacent l'emprise du sujet sur sa parole? Fondée sur l'analyse des représentations verbales du psychique, la psychanalyse a montré que la parole est l'actualisation d'un langage mue par une force pulsionnelle. L'investissement pulsionnel dans le langage vient élargir la définition de la parole, hors de sa dimension strictement intentionnelle. Pour le dire autrement: le sens excède désormais le signifié. Cependant, il y a une résistance de notre civilisation à ce débordement de sens, comme le précise de nouveau Lavie, puisque dans toute énonciation, la prééminence est toujours accordée "au contenu de ce qui est énoncé, au total détriment de la portée relationnelle de l'énonciation elle-même» (Lavie 1905/1988: 22). Que certains mots, qu'une certaine syntaxe surgissent à la place d'autres échappe à la majorité des auditeurs et des lecteurs attachés au sens des mots. À l'encontre de cette fascination du mot, le psychanalyste sait écouter et lire autrement. Ici commencent à apparaître les similarités entre la pratique du traducteur et celle du psychanalyste: les deux sont appelés à écouter et à organiser des signifiants selon un système de valeurs, mais qui ne préexiste pas à l'écoute. À la limite, le sujet traduisant «met au jour des signifiants fantasmés qu'il "reconnaît" dans l'original. [... L] e traducteur est prêt à trouver dans le texte de départ de quoi nourrir son investissement en même temps qu'il l'investit ${ }^{4}$ de ses affects» (Nouss 1998: 206). 
Dès lors qu'on veut penser la traduction par les moyens théoriques de la psychanalyse, on ne peut ignorer la mise en garde que Berman a formulée à cet égard, réservant aux analystes la compétence d'une telle approche, "pour autant qu'ils font l'expérience de la traduction comme d'une dimension essentielle de la psychanalyse elle-même» (Berman 1999: 50). Mais si les analystes sont les seuls habilités à parler de psychanalyse sur le terrain de la traduction, en revanche, ils n'ont pas su avancer une théorie du langage qui dépasse le dualisme de la théorie traditionnelle du signe, enfermée dans ce que Meschonnic rapproche d'une schizophrénie, du fait de la scission insurmontable entre les deux faces de l'entité: le signifiant et le signifié ${ }^{5}$ Quant à la linguistique de l'énonciation et ses travaux d'analyse du discours, elle est la mieux placée après tout pour fournir un cadre conceptuel alliant Saussure et Freud. Elle n'aura toutefois pas retenu les concepts de lalangue [sic], de parlêtre [sic], de signifiantmaître et de barre interne au signe que Lacan a avancés dans les années 1970 (Lacan 1975) : celui-ci en aura été quitte pour sa «linguisterie» (Lacan 1975: 29; Normand 2001: 23). Cette réticence théorique donne à comprendre que les linguistes ne sont pas disposés à imputer les trébuchements du sujet parlant aux mécanismes de l'inconscient et traiteraient comme autant de marques du sujet parlant répertoriables sous la rubrique générale des shifters les dérapages syntaxiques, lexicaux et phonologiques qui surgissent dans le discours. Au contraire, pour le psychanalyste, ces mêmes phénomènes se démarquent par leur caractère singulier et hétérogène, en ce qu'ils sont propres au sujet en train de parler, mais à la fois traversés par la parole de l'autre. Ainsi, la relation entre psychanalyse et linguistique se fait davantage sous le mode de la disjonction. Le point de divergence serait d'ordre épistémologique: alors que la linguistique se prête à l'étude du général, tentant par là de dégager les constantes sous-jacentes aux mécanismes communs du langage pour les formaliser, la psychanalyse, quant à elle, s'intéresse au " "détail singulier" d'une parole dans ses possibilités d'équivoque» (Normand 2001: 28) qui se joue dans l'usage le plus ordinaire de la langue et survient dans les "événements d'énonciation: lapsus, silence, refus de poursuivre, hésitation, répétitions sont depuis longtemps et quasi unanimement considérés dans la pratique psychanalytique comme focalisateurs d'interprétation » (Normand 2001: 169).

Éclairés par les travaux d'Authier-Revuz, qui allie la polyphonie bakhtinienne à la thèse de Lacan - selon laquelle l'être humain est produit par le langage davantage qu'il ne le produit -, certains linguistes de l'énonciation cherchent à interrompre ce dialogue de sourds, entre une linguistique rétive à l'Inconscient de la psychanalyse et une psychanalyse méconnaissant les avancées linguistiques depuis Saussure. Autour du thème Linguistique et psychanalyse, se sont réunis au colloque de Cerisy en 1998 des chercheurs ambidextres, maniant les outils de la linguistique dans l'étude des phénomènes singuliers et instables qui intéressent l'analyste. Leur point de convergence? La division de l'être humain en tant que sujet jamais pleinement maître de sa parole. Orale ou écrite, la parole est «ce qui, par-delà l'ordre du discours, subvertissant parfois l'ordre normée [sic] de la langue, voudrait à tout prix être dit » (Fenoglio 2001: 169). À la différence du discours, donc de la langue s'actualisant en fonction des paramètres particuliers que sont la situation, le contexte et la performance du locuteur, la parole, elle, est infléchie par une irruption tout à fait aléatoire et singulière du déroulement linéaire du discours. Plus près de nous, Venuti (2002: 230) aborde l'inconscient en traductologie, proposant de porter une attention particulière 
au faux ami, qui peut être révélateur d'un désir inconscient et qu'il faut alors prendre comme un lapsus.

\section{Lapsus translatandi}

Les irruptions surviennent dans l'énonciation sous forme d'actes manqués et surgissent fréquemment dans la pratique du traducteur par les lapsus d'écriture et de lecture, la perte, l'oubli et l'erreur momentanée, phénomènes que nous regroupons sous la catégorie générale des lapsus translatandi. La typologie des lapsus établie par Freud est ici reprise, mais adaptée à la dynamique traductive. Au nombre de ceux-ci, surviennent plus spécifiquement les lapsus calami (lapsus d'écriture), qui ont ceci de particulier qu'ils se commettent doublement: ils surgissent et ne sont pas relevés à la relecture des textes.

1) l'interversion de mots, comme il arrive souvent dans le cas des déterminants juxtaposés abondants en anglais et que le traducteur copie en français (p. ex. : chaise de secrétaire verte pour green secretary chair);

2) l'empiètement d'une lettre ou d'un mot sur un autre, ce qui entraîne une faute d'orthographe à la barbe du correcteur automatique (p. ex.: vingt terreurs d'écriture) ou encore un doublon;

3) la confusion par contraction ou condensation de morphèmes (barbarisme) ou de propositions (solécisme);

4) la substitution d'un mot par un autre, ce qui amène à dire tout le contraire de ce qu'on voulait. Les contresens en sont des produits éloquents, mais il y a aussi les dates et les noms propres mal reportés, qui ne manquent pas de surprendre.

Aux lapsus calami s'ajoutent les lapsus de lecture (lapsus lectionis), lesquels opèrent principalement par la substitution complète d'un mot par un autre qui se trouve dans son périmètre, souvent à la ligne au-dessus. En effet, il n'est pas rare de s'acharner quelques minutes à vouloir expliciter afin de le traduire le non-sens contrariant qui est généré par ce type de lapsus, bien que le traducteur expérimenté finisse par disqualifier la proposition insolite et relise la phrase en la suivant du doigt comme un troisième œil pour se rendre compte, soulagé, de sa méprise.

Viennent ensuite les autres actes manqués: la perte ainsi que l'oubli et l'erreur momentanés. La perte se caractérise par le fait qu'on s'en étonne et s'en trouve contrarié au lieu de la trouver incompréhensible. Elle se manifeste dans les textes sous forme d'omission, laquelle peut porter sur un signe diacritique, un mot, une phrase ou, plus gravement, sur un passage complet. L'omission est d'autant plus significative qu'elle résiste aux relectures du texte. Également digne d'intérêt, l'oubli momentané d'un nom propre, de mots étrangers, mais surtout du mot juste. Qui n’a pas déjà eu la sensation vive et agaçante d'avoir le mot sur le bout de la langue et d'en rester contrarié jusqu'à refuser tout substitut synonymique? Ce blocage, vécu comme un passage à vide, est peut-être causé par l'angoisse du traducteur devant le constat sans cesse renouvelé qu'il est seul devant un vaste choix de mots ou de tournures syntaxiques (Chukwu 1991: 562). Le dernier type d'acte manqué est l'erreur momentanée, ou la méprise, «comme lorsqu'on croit pendant quelque temps à des choses dont on savait auparavant et dont on saura de nouveau plus tard qu'elles ne sont pas telles qu'on se les représente» (Freud 1922/1961: 15). Ainsi, les faux sens flagrants surgis de faux amis, devant lesquels nous ne pouvons que nous étonner après coup lorsqu'ils nous sont signalés (mont de Vénus pour Mount of Venus). 
Il importe de souligner que les lapsus de traduction, au même titre que les lapsus de parole spontanée, ont ceci de particulier qu'ils sourdent hors de la maîtrise du locuteur de manière imprévisible, percutante et contrariante, telle une échappée de parole singulière. S’il est vrai que les événements d'énonciation surgissent souvent d'une occasion morphologique, sémantique ou syntaxique analysable après coup d'une perspective linguistique, ils ne sauraient pourtant pas s'y réduire. De fait, Freud (1922/1961: 32) les considère non pas comme «des accidents, mais des actes psychiques sérieux, ayant un sens, produits par le concours ou, plutôt, par l'opposition de deux intentions différentes». En guise d'exemple, nous tenons à relater un cas éloquent de contresens né d'une conjoncture morphologique dont nous avons été témoin. Une vingtaine d'apprentis traducteurs disposaient de deux semaines pour traduire un texte de nature commerciale intitulé Underserved Customers. Au moins la moitié a rendu le titre par Clients immérités, ce qui traduit Undeserved Customers. Tout comme ces apprentis, vous aurez lu vite et aurez passé outre la présence du $r$ dans l'adjectif du titre. Ce n'est pas tant d'avoir mal lu qui est curieux ici, puisque les mots sont identiques à une lettre près, mais plutôt qu'on s'acharne à affubler le texte d'un titre qui ne résume en rien le contenu du texte. Comment expliquer que les étudiants aient pris le contre-pied du texte qui pourtant traitait en détail de la clientèle négligée par les entreprises qui n'adaptent pas la commercialisation de leurs produits à sa situation linguistique? Bien qu'il ne nous appartienne pas de conjecturer sur l'origine de cette échappée de parole, il est tentant d'avancer qu'inconsciemment une résistance au mercantilisme effréné de l'économie pressait de se faire entendre. Que connaissons-nous au fond des motivations du sujet traduisant? Les traductologues tenants de la sociologie ont trouvé leur réponse dans la thèse bourdieusienne selon laquelle le traducteur, animé par un désir de reconnaissance, d'autorité sociale et de prestige culturel, choisit un texte dont l'auteur jouit déjà d'un capital symbolique afin de se substituer à lui. À son insu donc, le traducteur tendra à résister au texte, ce qui l'amènera à omettre des éléments et à commettre des contresens dans le but précisément d'outrepasser la figure de l'écrivain (Venuti 2002: 232). Cependant, l'explication sociologique du désir du traducteur se limite à l'économie du texte littéraire et ne rend pas compte des textes pragmatiques anonymes qui ne présentent aucun potentiel de gain en capital symbolique. Pratique linguistique, pratique sociale, la traduction doit être envisagée comme une activité de lectureécriture traversée par la parole singulière du sujet traduisant. L'attention portée à la singularité de l'énonciation répond au souhait de Peraldi, qui espérait voir se constituer une «science des singularités, une science de la parole, la linguistique n'étant que science de la langue» (Peraldi, date inconnue).

\section{Pas sans Peraldi}

Émigrant à Montréal, Peraldi quitte la France et commence à exercer en 1973 à l'hôpital psychiatrique Douglas. Il y mène des psychanalyses institutionnelles, mais de manière peu orthodoxe, puisqu'il laisse aux patients un espace de parole égal à celui du personnel soignant. Ce fonctionnement démocratique des séances aura tôt fait de soulever un conflit d'autorité auprès des psychiatres-administrateurs, qui remercièrent Peraldi. À la fermeture forcée des séances d'analyse à l'hôpital, Peraldi tiendra la psychiatrie dans son collimateur et s'attachera de 1976 à 1981 à critiquer amèrement 
le pouvoir des psychiatres sur la maladie mentale ${ }^{6}$. Dans une optique althussérienne, étayée par les thèses de Foucault et de Deleuze et Guattari sur la folie et le capitalisme, Peraldi met en cause les lieux de cette «pan-psychiatrie nouvelle (psychologues, travailleurs sociaux, infirmiers psychiatriques, thérapeutes en tous genres voire même bon nombre de psychanalystes [...] tous unis sous la férule du psychiatre et de son administration de la santé mentale)» (Peraldi, date inconnue). Il déplore ses moyens (la médicalisation de la folie et le refus de la mort) et ses intérêts (la perpétuation de l'idéologie bourgeoise par le maintien des conditions de production capitaliste). Mais si Peraldi en a contre la psychiatrie, c'est pour protéger la spécificité du savoir et du savoir-faire psychanalytiques. Reconnaissant à la psychiatrie et à la psychanalyse des aires épistémologiques différentes, il affirme que la psychanalyse est forte du nouvel esprit scientifique posé par Bachelard dans sa manière novatrice de penser l'être humain depuis Freud. À ce titre, elle rompt radicalement avec le rationalisme positiviste et empirique qui domine la psychiatrie et la psychologie. Même s'il affirme haut et fort la spécificité et la légitimité de la psychanalyse, Peraldi n'hésite pas à pester contre le «fait qu'au Canada la psychanalyse - rongée par la tentation médicale - est plutôt frappée d'aphasie [...] et rejetée, au pire, par nombre d'intellectuels du côté $[. .$.$] d'une sous-spécialité psychiatrique [...] ]^{7}$ (Peraldi 1985: 177). Impossible ici de ne pas faire le parallèle entre le constat du statut ancillaire de la psychanalyse (qui n'est «que l'une des servantes de la psychiatrie» [dans Reik 1975: 116], que déjà Freud déplorait dans une lettre à Reik datée du 3 juillet 1938, et le constat d'annexion de la traduction par la linguistique ${ }^{8}$. Dans son ouvrage Théories contemporaines de la traduction, Larose qualifie d'inféodation ce rapport de force épistémologique, référant au traitement que Fédorov réservait à la traduction, dominée par le mode linéaire et exhaustif de l'analyse axée sur le mot ou la phrase et caractérisée par l'étude de problèmes isolés et la génération de règles universelles.

Navré de ce qu'il qualifie d'abâtardissement des thèses freudiennes en Amérique du Nord, Peraldi en dénonce l'interprétation neutralisante qu'il taxe de surcroît de déviationnisme et de réductionnisme, à commencer par l'évacuation de la pulsion de mort, qui fonde le concept de l'Inconscient. Aussi, il exhorte ses pairs à critiquer la psychanalyse nord-américaine, à la déconstruire, à la contester, à la déterritorialiser, à la détruire et à la distribuer à tout le monde (Peraldi 1977: 287, 290). «Avec l'introduction de la pulsion de mort, Freud introduisait une conception plurielle du sujet. Contre le "Je suis UN" [...]» (Peraldi 1977: 289). Cependant, chez les psychanalystes états-uniens des années 1950, la ego psychology a réuni toute une école de pensée revendiquant un moi fort pour le sujet, qui serait uni donc non divisé, prétention tant décriée par Lacan, qui la disait fonction de méconnaissance. Lanalysé ${ }^{9}$ n'avait qu'à s'identifier au moi fort de l'analyste pour retrouver la maîtrise de son Ego: "l'analysé purifié, transformé, devenu "conscient" de ses difficultés, est un "sage”. Merveilleuse publicité? Illusion naïve?» (This et Thèves 1982: 44). Or selon Lacan, le moi parcourt un chemin étroit, sa liberté d'action étant contrainte par les deux pôles qui l'écrasent: le contrôle de l'ordre symbolique et l'ingérence incontrôlable de l'inconscient. Les psychanalystes de l'ego américains réfutent la soumission du moi à l'inconscient, forts de l'idée constitutionnellement fondée de liberté et volonté d'action (Leupin 2004: xvi). Pour Peraldi, ardent défenseur des travaux de Lacan, l'identification au moi fort aliène le sujet, ruinant l'avènement d'un sujet pulsionnel qui passe par la parole de l'analysant, par une combinatoire pulsionnelle 
particulière des signifiants que l'analyste devait écouter et reconnaître. Tabler sur le moi idéal, c'est aussi reconduire et renforcer chez le malade les structures de l'idéal du moi, ces modèles auxquels le sujet, poussé par le narcissisme de son désir, cherche à se conformer, notamment par l'activation des mécanismes du refoulement, de la culpabilité et de la conscience.

En fait, il est intéressant de souligner que la pulsion de mort (Todestriebe) est arrivée en Amérique par le faux sens de sa traduction calquée de l'anglais death instinct, ce qui l'a d'emblée enfermée dans le paradigme biologique. Mais la biologie, dont relèvent la médecine et sa branche psychiatrique, s'occupe des organismes vivants et, conséquente avec elle-même, a déclaré l'instinct de mort comme un postulat rédhibitoire; en tant que science du vivant, elle aurait été en échec de reconnaître au nombre de ses axiomes que le corps fût programmé à mourir. Aujourd'hui, la science neurobiologique admet le potentiel latent de mort inscrit dans le bagage génétique, depuis qu'elle sait «que toutes nos cellules possèdent le pouvoir à tout moment de s'autodétruire en quelques heures " (Ameisen cité dans Green 2002/2005: 349). À preuve, le suicide cellulaire, ou l'autodestruction de la cellule, empêche la prolifération de cellules malignes propre à l'évolution du cancer. Ainsi, la pulsion de mort - non pas l'extinction de la matière, mais la force de mort - se présentait comme un concept révolutionnaire de la théorie freudienne, mais qui s'est trouvée neutralisée et donc désamorcée aux États-Unis.

Vaille que vaille, Peraldi a lutté contre le «psychanalysme» (Peraldi, date inconnue), l'état dégradé de la psychanalyse qui, de la dialectique entre pratique et théorisation que Freud lui souhaitait, a été réduite à un programme instrumental et normatif axé sur la visée adaptative de la cure psychanalytique, c'est-à-dire le rétablissement des écarts comportementaux concordant à une structure de la personnalité établie comme étant normale. Pour Peraldi, normalité, norme, assujettissement et exploitation capitaliste sont solidaires et complices du pouvoir d'une élite financière puissante. On reconnaît son allégeance marxiste, le moteur de la critique poststructuraliste qui a généré entre autres objets de pensée critique la théorie matérialiste du sens. Critique du pragmatisme dédaigneux de théorisation symptomatique de l'Amérique du Nord, Peraldi défend la psychanalyse en tant que pratique théorique. Autant en psychanalyse qu'en traduction, Peraldi (1982b: 10) se plaint que l'activité réflexive soit abaissée à l'état de « construction gratuite et superflue (intellectualisante dit-on non sans quelque mépris) réservée aux seuls pontes institutionnels pour se représenter ce qu'ils font en termes plus ou moins sophistiqués». Le même pragmatisme qui fait dire que les textes de Lacan sont illisibles. Cette intolérance pragmatique sévit aussi en traductologie, de sorte qu'en Amérique du Nord l'œuvre magistrale de Meschonnic est peu lue et encore moins traduite. Et cela, malgré le fait qu'il s'impose en France comme une figure marquante de la théorie littéraire par la constance d'une réflexion qui se fait depuis plus de 30 ans sur la traduction littéraire. Pym (2003: 338), qui en a traduit quelques extraits, dont notamment les «Propositions pour une poétique de la traduction», tirées de Pour la poétique II, souligne la réticence des universitaires anglophones à discuter ses travaux.

À propos de Meschonnic, on notera, mais très brièvement, que certaines de ses idées résonnent vivement avec celles de Peraldi. Les deux soulignent à maintes reprises la nécessité d'interdépendance entre la pratique et la théorisation, vers une théorie matérialiste. Comme nous l'avons précisé, Peraldi défend la pratique théorique 
de la psychanalyse qui à son avis ne s'oppose pas à la clinique, mais au contraire la complète. Quant à Meschonnic, il a travaillé pour une poétique de l'écrire et du traduire, selon laquelle « [s] eul l'empirique est le lieu de la théorie. Il n'y a que là qu'elle se fait et se défait» (Meschonnic 1989: 98). Chez les deux théoriciens, théorie et pratique sont consubstantielles, parant ainsi contre l'empirisme soumis à la tyrannie des lieux communs, des idées arrêtées et des théories dominantes. Là où l'empirique trouve des questions, l'empirisme cherche des réponses. Sur le plan épistémologique, les positions critiques de Peraldi et de Meschonnic se rejoignent également: le psychanalyste critique la médicalisation de la maladie axée sur la suppression des écarts comportementaux et le traducteur déplore la naturalisation-neutralisation des textes par la réduction des écarts à la norme linguistique. Tandis que le premier met en cause l'impérialisme thérapeutique de la psychiatrie, le second dénonce l'impérialisme théorique de la linguistique, qui plaque une méthodologie scientifique ellemême calquée sur les sciences exactes. Peraldi et Meschonnic critiquent le statut ancillaire de leurs champs d'activité, perçus comme des sous-disciplines relevant d'une science en position d'autorité. Il est intéressant de noter que les deux, portés par une verve subversive, marquent grammaticalement les rapports de force entre des concepts compromis à l'ordre dominant et leurs corollaires critiques. Ainsi, le suffixe péjoratif -isme sert à exprimer la déviation et la réduction d'un concept ( $p s y$ chanalysme chez Peraldi; empirisme et historicisme chez Meschonnic). À l'opposé, leurs pendants psychanalyse, empirique et historicité recèlent une charge positive, en tant qu'ils sont dynamiques, productifs et surtout critiques du pouvoir et des idées reçues.

Une autre critique que Peraldi adresse à la psychiatrie porte sur l'incompétence thérapeutique de celle-ci, qui s'acharne à vouloir guérir les psychoses - schizophrénie et paranoïa - au sens que la médecine occidentale entend guérir, c'est-à-dire faire disparaître. La plus grande faute dont Peraldi accuse l'appareil psychiatrique largement médicalisé est son défaut d'écoute. De ce fait, la psychanalyse doit combattre le pouvoir répressif médical en créant un espace d'écoute ${ }^{10}$. Selon Peraldi, le défaut d'écoute touche aussi le traducteur, formé pour normaliser ses productions écrites en fonction de ce qu'il est convenu d'appeler le bon usage et le français standard.

\section{Le corps de la parole dans le langage}

Traducteur et psychanalyste doivent pouvoir lire autrement. Même si on parle couramment d'écoute, Freud propose de lire le récit du rêve ou des symptômes du patient au pied de la lettre, comme un texte. De même, en ses qualités de lecteur hors pair, le traducteur sait très bien que «le langage fait quelque chose en même temps qu'il dit. Il ne fait pas nécessairement ce qu'il dit» (Meschonnic 1982: 83). Le psychanalyste et le traducteur ont ceci en commun qu'il leur appartient d'élaborer avec patience et précaution les lois qui président à l'ordonnancement du signifiant (Peraldi 1982b : 25), ce qui implique de demeurer sensible aux forces pulsionnelles que le corps textuel éveille chez le corps traduisant. Pour le dire autrement, il faut prêter attention à la matérialité et à l'interaction des mots et des marques textuelles sans en connaître d'avance la valeur. Allitération, prosodie, champ lexical, monosyllabisme, mouvement syntaxique, répétition, ponctuation, etc. sont autant de matériaux signifiants que le lecteur peut investir de sens. Sans ce travail sans cesse renouvelé, le corps 
traduisant est coupé de sa dynamique pulsionnelle, ce qui entraîne une perte grave, tel que Peraldi l'explique:

[...] le langage participe de la vie pulsionnelle longtemps avant de servir à la communication sociale qui exigera le refoulement de cette fonction première [qui sert à établir la relation entre l'enfant et sa mère par la répétition du matériau sonore]. C'est pour cela que sa perte dans la traduction n'est pas un simple détail mais le geste le plus grave qu'on puisse commettre sur le langage, la perte la plus sinistre que l'on puisse faire subir à tout sujet: la perte du pulsionnel (Peraldi 1985: 185).

Le défaut d'écoute en traduction résulte de la censure de l'oreille, que nous proposons de nommer otocensure. Le jeu de mots évoque l'oreille par le préfixe oto-, qui donne à entendre auto-, pour exprimer la censure de soi et de l'écoute. Dans ce double barrage, le sujet traduisant ne se laisse pas aller à entendre la matière sonore du sens, habituellement maintenue dans la secondarité accessoire du signifiant. Le plein poids du propos de Peraldi se fait sentir dans les traductions littéraires insipides, ces textes traduits de manière hyperfluide qui laissent le lecteur indifférent. Double perte pestait Peraldi, et pour le langage, et pour le sujet. Alors que la traduction s'évertue à se conformer aux normes du bon français écrit, l'original, lui, se joue des contraintes de la correction linguistique, parce que c'est dans la transgression des limites que se joue le pulsionnel, les traductions normalisantes banalisent et appauvrissent la texture textuelle. Il en résulte un texte qui ne fait rien sauf se conformer au canon esthétique du français, axé sur la clarté, la linéarité et la fluidité, dont Berman (1999: 49-68) a jaugé la force déformante qui informe à un niveau inconscient le travail du traducteur.

Moment crucial d'activation d'un sens, la lecture engage le sujet traduisant dans une activité d'écoute du corps textuel. Prêter l'oreille au signifiant corrobore le constat de Freud quant à la nature rigoureusement structurée et donc analysable de celui-ci, et ce, par rapport au signifié flou et polysémique. L'érotique selon Peraldi tend vers une sémantique pulsionnelle, suivant les travaux de Kristeva (1974) fondés sur les bases pulsionnelles de la phonation de Fónagy (1971): «[...] écouter, lire, implique qu'on laisse résonner en nous sur le mode pulsionnel ces textes où l'insistance pulsionnelle se manifeste par l'insistance sonore d'un certain groupe de phonèmes qui domine telle ou telle zone du texte» (Peraldi 1985: 186). Ainsi, les bilabiales [b], [p], [m] et les dentales [d], [t] correspondraient à une pulsion orale, les occlusives postérieures $[\mathrm{k}],[\mathrm{g}]$ à une pulsion anale, les fricatives et les sifflantes [f], [s] à une pulsion urétrale et l'apicale $[\mathrm{r}]$ à une pulsion génitale.

À y regarder de plus près, la sémantique pulsionnelle vient contredire l'idée de laisser résonner en nous les textes, car sa démarche revient à l'application d'une grille de lecture, ou d'écoute, établie a priori. Elle neutralise le potentiel heuristique d'une écoute chaque fois neuve de la matérialité sonore du sens, puisque le traducteur aurait déjà une idée préconçue de ses valeurs sémantiques. Par exemple, faire correspondre systématiquement la dominance de [s] à une tension urétrale - le [sssss] de pisser, pour faire dans le familier - reviendrait à lui attribuer d'office une charge pulsionnelle prédéterminée au détriment d'autres possibilités de signifiance. La contradiction entre le polymorphisme sémantique et l'univocité du son désamorcerait la sensibilité du corps érotique que Peraldi tente d'éveiller. Au demeurant, cette sémantique pulsionnelle négligerait l'accentuation, notamment l'accent de mots (comme l'espagnol et l'anglais) ainsi que l'accent prosodique propre au français (Meschonnic 1982: 668). 
Les voyelles non plus ne vaudraient rien. S’en trouverait corroboré, mais à l'avantage du signifiant cette fois-ci, le primat d'un sens prédéterminé que suppose la théorie du signe, du fait qu'elle accorde d'office la préséance au signifié. Les valeurs du texte seraient connues d'avance et il n'y aurait qu'à les repérer mécaniquement. La sémantique pulsionnelle n'est donc pas à prendre comme une grille d'écoute, mais plutôt comme une piste vers un mode de faire sens sensuellement, donc ailleurs que dans le signifié et autrement que par la fonction strictement communicative du langage. Chaque fois que l'ordre symbolique de la communication pose péremptoirement la question Qu'est-ce que ça veut dire?, il faudrait laisser la fonction érotique demander Qu'est-ce que ça (me) fait? En fait, l'apport de la psychanalyse aux sciences humaines aura été d'élargir le concept de sens hors de la fonction de représentation du langage et d'avoir donné cours aux potentialités de ce que parler peut faire, notamment affirmer la présence au monde du sujet parlant. Devant un texte, cette affirmation est celle du traducteur.

Investissant dans certains signifiants du texte, le corps traduisant en construit la valeur et sort ainsi radicalement de l'ordre dualisant du signe linguistique, qui limite le sens à une face de sa structure signifiant/signifié et, par le fait même, rend le sujet traduisant incapable de voir ni d'entendre du sens ailleurs que dans le signifié. À la nécessité d'élargir le sens, Meschonnic (1973a: 34) a proposé la forme-sens, qu'il incorporera plus tard dans l'idée de rythme. Le trait d'union traduit l'unité dialectique de deux concepts que la définition sémiotique oppose. La sémantisation d'un texte peut inclure des formes-sens, telles que les rimes, les blancs typographiques, les rapports entre voyelles et consonnes, les relations syntagmatiques et paradigmatiques, la syntaxe, les accents prosodiques... Le signifiant peut être tout ça, puisque dans son participe présent substantivisé, il désigne toute chose en train de signifier (Meschonnic 1982: 70). La rigueur des travaux de Meschonnic sur «l'agir du langage» (1999: 140) devrait nous avoir convaincus que le sens ne réside ni uniquement ni sûrement dans les mots, dont l'étymologie garantirait la vérité. Quant à la vérité, elle «est peu de chose en regard de la persuasion que nous recherchons en parlant»(Quignard 1987: 15).

Si le sens est à voir, à entendre et à faire partout à partir du matériau textuel, cela ne se réalisera que si les sens physiques du corps traduisant sont éveillés, que si le corps érotique dont parle Peraldi est mis en activité pour traduire non plus ce que les mots disent (tel qu'on chercherait à décoder l'intention de l'auteur), mais ce que les mots font (Meschonnic 1999: 138). Comme l'a répété Freud pour la lecture des rêves, aucune clef ni grille ne préexiste à l'interprétation des contenus psychiques, qui viendrait dicter le sens des images visuelles et sonores. Ainsi, le traducteur et l'analyste se trouvent sur les mêmes bases méthodologiques: le sens ne préexiste pas au texte, ni aux symptômes, ni aux rêves. Les réseaux de signifiants se construisent différemment, selon la valeur dont les investira le corps traduisant. Lorsque celui-ci lit un texte, il en organise les valeurs selon sa situation historique, transformant ainsi les valeurs linguistiques du texte en valeurs de son discours. Nous ne faisons là que rappeler la fonction déictique qu'Émile Benveniste accordait au langage toujours dépendant dans son actualisation de variables historiques, culturelles, psychologiques, etc., qui rendent toute production de sens unique, qu'on lise, écrive ou traduise. À cette liste, il faudrait rajouter la variable physique sur laquelle Peraldi a misé et Meschonnic tout autant, c'est-à-dire l'oreille par laquelle passe l'écoute des singularités du texte. Dans 
leurs champs respectifs, ces praticiens ont constaté combien réprimée se trouvait la faculté d'écoute de la matière sonore. Cette otocensure résulte soit du surinvestissement conditionné du signifié, soit de l'atrophie ou de la désensibilisation de l'oreille. Dans les deux cas, le sujet traduisant se prive de la matérialité entendue des mots (Meschonnic 1973b: 258). En réaction à cette chute du corps, nous prenons appui sur le concept de corps érotique avancé par Peraldi (1985: 177) et proposons une érotique du traduire.

\section{L'érotique du traduire}

Réveiller la fonction érotique du corps traduisant nécessite qu'il se sache mu par trois forces: la sensibilité signifiante, les pulsions de vie dites érotiques et leur corollaire négatif, l'otocensure. La sensibilité signifiante du sujet traduisant correspond à sa capacité de faire sens par les sens de son corps, c'est-à-dire grâce à sa faculté d'éprouver des sensations physiques, notamment à la lecture d'un texte. Cette sensibilité doit passer par la sensibilisation du corps traduisant, donc nécessairement physique, à commencer par l'oreille. Le sujet traduisant doit se mettre à l'écoute de la matérialité sonore du texte à traduire pour lire et capter la singularité de sa structure combinatoire. Lire en écoutant et écouter en lisant, a dit Peraldi, afin de se laisser toucher par les mots-corps, les mots-sexes des autres fait appel à la fonction érotique du corps traduisant. Premier sens à se développer, l'ouïe préexiste aux autres sens chez le fotus, qui perçoit la voix de sa mère, sa respiration, les bruits de sa circulation sanguine et de sa digestion. Il entend bien avant de voir, avant de naître. L'être humain, sa vie durant, entend dans la parole bien au-delà de la signification des mots: le timbre, la tonalité et l'intensité de la voix, lesquels nous informent sur le locuteur. Pourquoi devant le matériau textuel le lecteur se coupe-t-il de ce potentiel de sémantisation sensuelle?

Il n'est pas exagéré d'affirmer que l'ouïe se trouve largement réprimée dans la pratique traduisante, malgré son pouvoir de décupler les manières de faire sens du texte à traduire. Mais comment peut-il en être autrement depuis les enseignements de l'École du sens: faire passer le signifié, s'éloigner du texte, quitter les mots, lire entre les lignes? Si cette démarche convient davantage à la traduction de textes techniques, en contrepartie, on sait que par habitude et efficacité, elle est devenue le lot de la traduction littéraire. À bien y penser, la répression de l'ouïe tient peut-être précisément de sa puissance heuristique. Pourtant, le psychanalyste a su, lui, faire travailler son oreille. C'est donc en empruntant à la praxis de l'analyste son mode d'écoute attentive aux manifestations physiques de la matière sonore que le traducteur se laissera toucher par le corps textuel, souhait qu'avait formulé Peraldi pour la traduction.

Étonnamment beaucoup se trouve condensé dans le verbe traduire, en théorie conçu de façon unitaire, mais en pratique se dédoublant entre lire et écrire. Lire amène à organiser les signifiants; écrire implique la réalisation et la mise à l'épreuve de cette organisation. Si à la lecture d'un texte nous en investissons certains éléments d'une valeur, l'enjeu majeur consiste à faire valoir en retour ces investissements. Il y a fort à parier que le moteur de ce retour est alimenté par la tendance du traducteur à vouloir conserver le texte original. Cette tendance suit le principe dynamique et fondamental de la pulsion de vie telle que la conçoit Freud dans son deuxième modèle de l'appareil psychique (le ça, le moi et le surmoi). 
Nommées aussi l'éros par Freud, les pulsions de vie poussent le sujet à établir des unités toujours plus grandes, but qui participe d'un principe de liaison. Liaison signifie ici à la fois l'idée d'une cohésion maintenue dans un ensemble et la fixation d'une quantité d'énergie qui ne peut plus s'écouler librement (Laplanche et Pontalis 1997: 224). L'établissement et le maintien des rapports que suppose l'éros envisagé dans la pratique du traduire résonnent profondément avec une visée éthique par laquelle sont établis et maintenus des rapports entre langue et langue, texte et texte, culture et culture... et corps et parole, aurait peut-être ajouté Peraldi. Le lien entre le corps à soi et la parole de l'autre passe par l'écoute. L'ouïe est un sens social du fait que l'être humain dépend de l'autre pour apprendre à parler: il faut d'abord entendre la parole pour savoir comment la produire (Wulf 2007: 679).

Nul doute que le défaut d'écoute ramène le traducteur aux formes figées, aux expressions consacrées, aux tournures correctes, visant à renforcer le caractère idiomatique de son texte. Le résultat? Une traduction neutralisée qui certes paraît bien, mais qui ne fait rien, sauf se conformer à l'ordre établi.

Écouter pour lire et lire pour écrire: aucune de ces activités n'échappe à l'otocensure. Fonction permanente relevant du processus plus général de défense, la censure est le corollaire négatif des pulsions de vie, que Freud nomme l'éros. Il a été question plus haut du refoulement en tant que principe défensif fondamental de l'inconscient, qui bloque le passage vers la conscience des matériaux psychiques afin d'éviter ce qui perturbe le principe de plaisir. Freud rattache la censure à l'ensemble d'opérations effectuées par un processus plus large de défense, occupé à réduire et à supprimer toute modification de l'appareil psychique risquant de déstabiliser l'intégrité et la constance du sujet. «Dans la mesure où le moi se constitue comme instance qui incarne cette constance et qui cherche à la maintenir, il peut être décrit comme l'enjeu et l'agent de ces opérations» (Laplanche et Pontalis 1997: 108). La constance du traducteur est mise en danger par la contradiction qui rend irréconciliable la ressemblance du texte traduit à la fois au texte de départ et aux autres textes de la langue-culture réceptrice (Venuti 2002: 237).

Vers l'autre versant du chiasme corps de la parole et parole du corps, nous voulons maintenant prêter attention à certaines échappées de parole singulière, telles qu'elles peuvent survenir sous la plume de traductologues.

\section{La parole du corps dans le langage}

Allouch (1982: 83) rappelle que « tout un chacun écrit bien au-delà de ce qu'il imagine écrire». Voilà une idée qui fait consensus en psychanalyse et qui touche la traduction en tant que pratique d'écriture. Le langage du corps ne s'oppose pas au langage verbal, le verbe n'étant qu'un moyen parmi d'autres d'expression (il y a les gestes, la voix), qui passe nécessairement par le corps (Meschonnic 1985: 131).

Utilisons le postulat psychanalytique selon lequel le sujet humain est produit par le langage davantage qu'il le produit et employons-le à la lecture du livre Deux langues, six idiomes ${ }^{11}$ de de Buisseret. Cet ouvrage-culte d'une certaine génération de traducteurs se démarque sur le plan discursif par la métaphorisation pathologique que l'auteure fait de l'état de la langue française en traduction. Elle fait travailler la métaphore de manière systématique dans son ouvrage, où elle diagnostique et recense les principaux troubles, maladies et symptômes dont souffrent les traducteurs, au 
nombre desquels l'hydropisie verbale, la cacographie chronique, la concrétite, la distortionnite, l'hypothésomanie, la culturite, l'hypo-attention et l'incompréhensite. Le premier chapitre s'intitule radicalement Le mal. Il y a là beaucoup d'humour et d'esprit. Il y a là aussi une fixation sur la pathologie dont témoigne la métaphore tératologique qui innerve puissamment le propos.

Très attachée à la langue française, de Buisseret entretient un rapport véritablement passionnel aux mots et s'emploie à maintenir celle-ci en bonne santé. Cet amour de la langue n'est pas platonique, comme peut le laisser croire le portrait qu'en fait Delisle, selon lequel «Irène de Buisseret chérit sa langue, la révère comme un amant sa bien-aimée ${ }^{12} »$ (Delisle 2002: 81). Son rapport à la langue est plutôt charnel, tel que nous en convainc le remède qu'elle propose à l'incompréhensite et qui consiste en l'immersion dans l'océan anglo-saxon afin «qu'il nous entoure, nous embrasse, nous étreigne, nous pénètre» (de Buisseret 1975: 144). Par ces verbes, la traductrice-rédactrice, réviseure, professeure de français chevronnée exprime vivement son rapport sensuel à la langue, qu'on ne connaît qu'à faire corps avec elle.

Laissons libre cours à la sensibilité du traducteur en ses qualités de lecteur hors pair, puisque nous l'avons vantée plus tôt, et glissons l'espace d'un paragraphe dans la lecture en abyme de ce que Delisle (2002) dit de ce que de Buisseret dit de la traduction dans le portrait qu'il a brossé d'elle. Nous constaterons qu'en contrepoint du discours somatique de celle-ci contraste le discours rationalisant de celui-là:

N’ayant pas à sa disposition un vocabulaire spécifique pour décrire les phénomènes de la traduction, Irène de Buisseret structure son manuel comme un traité médical. Dans une première partie, elle diagnostique le 'Mal': 1 . Hydropisie verbale; 2 . Cacophonie [sic] chronique; 3. Affections secondaires (Delisle 2002: 378).

Le terme facétieux cacographie est mal reporté sous la plume de Delisle et se transforme en cacophonie. Le lapsus sourd et annonce ce qui quelques lignes plus loin est confirmé par un commentaire ambivalent du traductologue, qui affirme à propos des images utilisées par de Buisseret (phrase enflée, phrase-ténia, traduction adipeuse, mot-éléphant, etc.) que "[s]i ce style imagé, ce vocabulaire métaphorique est dénué de toute valeur scientifique, il présente néanmoins l'avantage d'être clair et pédagogiquement efficace» (Delisle 2002: 378). Il n'en demeure pas moins cacophonique, mélange confus de plusieurs bruits ou voix pour Delisle, auteur de La traduction raisonnée, ce qu'il a laissé entendre malgré lui. Un acte manqué... si bien réussi!

Avide de montrer les «distorsions, déformations, faiblesses, maladies [...] ces déviations de la langue et du métier» (cité dans Delisle 2002: 382), de Buisseret donne à voir le corps de la langue comme un corps malade ou qu'un mal imminent guette. Sa métaphore pathologique est tant et si bien filée qu'elle se déroule sur des centaines de pages. Est-il exagéré de lire là la traduction du mal latent qui allait ronger la santé de cette traductrice passionnée? Est-ce du seul fait que de Buisseret ne disposait pas d'un métalangage qu'elle a recouru au paradigme du corps dans sa vitalité et sa dégénérescence ou s'agit-il d'une écriture somatique? On sait qu'elle a souffert d'un cancer, et nous présumerions sans doute trop en avançant que la maladie se tramait au moment où elle écrivait son manuel. Par ailleurs, la rédaction de celui-ci s'est échelonnée sur de nombreuses années au gré de notes compilées, d'observations cueillies sur le tas et d'exemples glanés, pour finalement être publié à titre posthume. Néanmoins, l'actualisation systémique du paradigme médical, surtout sur le mode 
de l'humour, résonne singulièrement et remarquablement. En clair, la métaphore filée de la pathogénique est si patente qu'elle traduit la maladie latente de l'auteure, malgré elle. En ce sens, Irène de Buisseret était écrite par ce mal qui la guettait; sa fixation au symptôme en disait bien plus que ce qu'elle imaginait écrire. Elle annonçait son destin en page liminaire du manuel, citant avec humour l'apophtegme du docteur Knock: «Tout homme bien portant est un malade qui s'ignore.» Et elle persiste avec une lucidité prémonitoire troublante: «Mauvais, médiocres, bons ou excellents, nous [traducteurs] sommes tous malades, tous contaminés à des degrés divers. Je le suis probablement plus que les autres; mais, l'âge aidant, je ne l'ignore plus» (De Buisseret 1975: 1). Ce qu'on peut appeler ici un excès de sens, tous ne seront pas prêts à le reconnaître. On ne peut nier, toutefois, qu'Irène de Buisseret se jettera dans le canal Rideau.

\section{Jalons}

Verbe, verve, vivre; voilà nos trois jalons et les trois vecteurs qui parcourent la psychanalyse et la traduction et par lesquels, de François Peraldi à Irène de Buisseret, en passant par Henri Meschonnic, il est possible de circuler. Faire entrer la psychanalyse dans la traduction permet d'envisager une autre dimension de l'activité traduisante, de l'ordre du pulsionnel. Passant Peraldi, transmettant ses idées, nous avons posé les bases d'une érotique du traduire qui amène la pratique sur le lieu du corps - le corps de la parole et la parole du corps dans le langage. Le langage sert à vivre, écrivait Benveniste. À faire (re)vivre les autres, ajouterons-nous, dans le droit fil d'une pensée benjaminienne de la traduction comme sur-vie.

Il n'y a rien de rassurant ni de plus contre-intuitif pour le corps traduisant que d'admettre que la parole ne reflète pas exactement ce qu'il veut lui faire dire parce qu'elle exprime autre chose qui lui échappe. La psychanalyse l'aura peut-être convaincu que parler, écrire, signifient d'abord convaincre de ce qu'il parle. Même lorsqu'il pense avoir refoulé sa présence jusqu'à se croire transparent, le corps traduisant finit toujours par se donner à lire, ce que font entendre les lapsus commis à son grand dam.

Et pourtant, c'est ainsi qu'il donne signe de vie.

\section{NOTES}

1. Peraldi a publié un triptyque sur l'écoute de la Folie, pour reprendre la majuscule qu'il faisait porter au vocable, peut-être pour en rejouer la stigmatisation ou encore pour en exacerber la présence: les deux articles qui suivirent s'intitulent Les lieux de l'écoute. Pour une clinique psychanalytique des psychoses et Les lieux de l'écoute. L'analyse institutionnelle.

2. Michel Peterson, directeur de la collection «Voix psychanalytiques» aux éditions Liber, travaille à la publication des séminaires de Peraldi, dont Le sujet. Séminaire 1981-1982 et L’Autre. Le temps. Séminaire 1982-1985. Quant au séminaire sur la mort, aux enseignements sur Lacan ainsi qu'aux autres écrits de Peraldi, Karim Jbeili les héberge sur son site: <http://www.calame.ca> (page consultée le 30 juin 2007).

3. Tels les articles respectifs d'Ingram (2001), de Venuti (2002) et de Basile (2005).

4. Quant au terme investissement, Jean Laplanche et Jean-Bertrand Pontalis (1997: 211) en donnent la définition suivante: "fait qu'une certaine énergie psychique se trouve attachée à une représentation ou un groupe de représentations, une partie du corps, un objet, etc.».

5. Pour une critique du dualisme du signe dont le schéma se perpétue dans les théorisations psychanalytiques, notamment celles de Nicolas Abraham, voir les pages 663 et 664 dans Critique du rythme (Meschonnic 1982). 
6. En réaction à cet acte coercitif des psychiatres-administrateurs, Peraldi publiera trois articles consécutifs dans Santé mentale au Québec: L'élangage de la folie (Peraldi 1978a), Les lieux de l'écoute: pour une clinique psychanalytique des psychoses (Peraldi 1978b) et Les lieux de l'écoute: l'analyse institutionnelle (Peraldi 1979).

7. Peraldi n'hésite jamais à monter au front épistémopolitique, et, pour prendre le pouls de sa parole, il faut relire cette phrase sans les points de suspension: «De leur côté les linguistes ne manqueront pas d'être horrifiés par l'usage un tantinet lacanien que je ferais ici du signifiant, puisque c'est par le biais de ce que la psychanalyse a pu nous dévoiler de la composante pulsionnelle de la parole que j'entrerai dans le vif de mon sujet, bien au fait qu'au Canada la psychanalyse - rongée par la tentation médicale - est plutôt frappée d'aphasie - ce qui ne serait pas, je pense, pour déplaire à Jacques Derrida - et rejetée, au pire, par nombre d'intellectuels du côté de l'escroquerie pure et simple lorsque ce n'est pas - ce qui ne vaut guère mieux - du côté d'une sous-spécialité psychiatrique - objet - quoi qu'il en soit - de la même méprise, du même mépris, de la même haine» (Peraldi 1985: 177). À la fois longue et hachée, cette phrase paragraphe est criblée de flèches autant dans le propos que typographiquement par la grêle de tirets. Autre fait intéressant, le conditionnel «ferais» dès l'entrée en matière de la phrase: un lapsus?

8. Mais cette annexion est chose du passé et la traductologie s'est affranchie de la linguistique... on y croirait presque, n'eût été la prédominance des linguistes composant le comité subventionnaire fédéral, représenté à six contre un traductologue en 2009. Pourtant, le comité 5 porte le nom Linguistique, linguistique appliquée et traduction, <http://www.sshrc.ca/SITE/about-crsh/committees-comites/ standard_research-ordinaire_recherche-fra.aspx\#a5> (page consultée le 5 août 2009).

9. Il semble que Peraldi montre l'opposition entre la relation analyste-analysant de la psychanalyse et la relation analyste-analysé de ce qu'il appelle le psychanalysme. En jouant sur les catégories grammaticales, il fait passer l'analysant du participe présent, engagé dans l'expérience analytique, au participe passé (forme passive du patient), afin de montrer clairement la passivation de celui-ci, son assujettissement à l'analyste.

10. Cependant, la médecine ne veut pas ou ne sait pas écouter la parole du malade et s'acharne à vouloir traiter le symptôme en le faisant disparaître par des moyens médicamenteux. Dans l'économie de la non-écoute aujourd'hui, un médecin pourra prescrire en moins de 15 minutes des antidépresseurs, et aura fait pour le compte des pharmaceutiques un autre client fidèle à la cosmétique des humeurs courant le circuit de la sérotonine, pour reprendre les mots percutants de Christian SaintGermain (2007) dans l'allocution Comprimés ou honoraires qu'il a prononcée à l'ouverture du colloque Transfert et addiction, tenu au Centre de recherche interuniversitaire sur la littérature et la culture québécoises, à l'Université de Montréal le 18 mai 2007.

11. Lauteure de cet ouvrage phare était chef de la traduction à la Cour suprême du Canada et a enseigné la traduction à l'Université d'Ottawa, entre autres emplois, par ailleurs nombreux et variés qu'elle a occupés.

12. Quant à la métaphore de l'amant (au masculin), il faut bien avouer qu'elle déconcerte quelque peu. Sur la question du genre, il y a encore lieu de se demander pourquoi l'auteur du portrait d'Irène de Buisseret refuse la féminisation des titres d'emplois qu'elle a occupés, tels que «réviseur et professeur adjoint», alors qu'il fait porter la marque du féminin à "traductrice-rédactrice» (Delisle 2002: 341). Cette décision semble d'autant plus singulière que Jean Delisle fait d'Irène de Buisseret une «comtesse de la traduction» dans le titre de son article.

\section{RÉFÉRENCES}

Allouch, Jean (1982): De la translittération en psychanalyse. Meta. 27(1):77-86.

BAsile, Elena (2005): Responding to the Enigmatic Address of the Other... New Voices in Translation Studies. 1:12-30. Consulté le 8 juillet 2009, <http://www.iatis.org/newvoices/ issues/2005/basile-NV2005.pdf>.

Berman, Antoine (1999) : La traduction et la lettre ou l'auberge du lointain. Paris: Éditions du Seuil.

Cнuкwu, Uzoma (1991): Plaidoyer pour la part de la jurisprudence en traductologie. Meta. 36(4):558-566.

DE Buisseret, Irène (1975): Deux langues, six idiomes. Ottawa: Carlton-Green.

Delisle, Jean (2002): Irène de Buisseret, «comtesse» de la traduction, pédagogue humaniste. In: Jean Delisle, dir. Portraits de traductrices. Ottawa: Les presses de l'Université d'Ottawa, 369-402. 
Ducharme, Jean-François (2005): Une exploration de l'ouvre de François Peraldi. In: Danielle Monast et Gilles Chagnon, dir. Voix, legs, parcours d'un psychanalyste. Montréal: Liber, 11-30.

Fenoglio, Irène (2001): Les événements d'énonciation: Focalisateurs d'interprétation psychanalytiques, matériau pertinent de l'analyse linguistique d'énonciation. In: Michel Arrivé et Claudine Normand, dir. Linguistique et psychanalyse. Paris: In Press Éditions, 167-184.

FóNAGY, Ivan (1971): Les bases pulsionnelles de la phonation. Revue française de psychanalyse. 35(4):543-591.

FreUd, Sigmund (1905/1988): Le mot d'esprit et sa relation à l'inconscient. (Traduit par Denis Messier). Paris: Éditions Gallimard.

FreUd, Sigmund (1922/1961): Introduction à la psychanalyse. (Traduit par Samuel JANKéLÉViтch). Collection Petite Bibliothèque Payot. Paris: Éditions Payot.

Gavronsky, Serge (1977): The Translator: From Piety to Cannibalism. Substance. 16:53-62.

Green, André (2002/2005): Idées directrices pour une psychanalyse contemporaine. Paris: PUF.

Grenier, Louise (2002): La folie à corps perdu. Le Coq-Héron. 169:137-144.

Jbeili, Karim (2005): Les aiguillages de la transmission. In: Danielle Monast et Gilles Chagnon, dir. Voix, legs, parcours d'un psychanalyste, Montréal: Liber, 93-102.

Ingram, Susan (2001): Translation Studies and Psychoanalytic Transference. TTR. 14(1):95114.

Kristeva, Julia (1974): La révolution du langage poétique. Paris, Éditions du Seuil.

LaCAN, Jacques (1975): Livre XX. Encore: 1972-1973. Paris: Éditions du Seuil.

Laplanche, Jean et Pontalis, Jean-Bertrand (1997): Vocabulaire de la psychanalyse. Paris: PUF.

Lavie, Jean-Claude (1905/1988): Les mots en jeu. In: Sigmund Freud. Le mot d'esprit et sa relation à l'inconscient. (Traduit par Denis Messier). Paris: Éditions Gallimard, 9-31.

Leupin, Alexandre (2004): Lacan Today. Psychoanalysis, Science, Religion. New York: Other Press.

Meschonnic, Henri (1973a): Pour la poétique II. Épistémologie de l'écriture. Poétique de la traduction. Paris: Éditions Gallimard.

Meschonnic, Henri (1973b): Pour la poétique III. Une parole écriture. Paris: Éditions Gallimard.

Meschonnic, Henri (1982): Critique du rythme. Lagrasse: Éditions Verdier.

Meschonnic, Henri (1985): Les états de la poétique. Paris: PUF.

Meschonnic, Henri (1989): La rime et la vie. Lagrasse: Éditions Verdier.

Meschonnic, Henri (1999): Poétique du traduire. Lagrasse: Éditions Verdier.

Michaud, Ginette, dir. (1998): Psychanalyse et traduction. TTR. 11(2):9-37.

Normand, Claudine (2001): Linguistique et/ou psychanalyse: de leur relation si elle existe. In: Michel Arrivé et Claudine Normand, dir. Linguistique et psychanalyse. Paris: In Press Éditions, 17-30.

Nouss, Alexis (1998): La traduction mélancolique (sur Paul Celan). TTR. 11(2):199-231.

Peraldi, François (1977): La psychanalyse nord-américaine. Philosophiques. 4(2):287-291.

Peraldi, François (1978a): L'élangage de la folie. Santé mentale au Québec. 3(1):1-17.

Peraldi, François (1978b): Les lieux de l'écoute. Pour une clinique psychanalytique des psychoses. Santé mentale au Québec. 3(2):1-26.

Peraldi, François (1979): Les lieux de l'écoute. L'analyse institutionnelle. Santé mentale au Québec. 4(1):40-61.

Peraldi, François (1982a): Lecture psychanalytique et traduction du texte littéraire. Meta. 27(1):126-128.

Peraldi, François (1982b): Psychanalyse et traduction. Meta. 27(1):9-25.

Peraldi, François (date inconnue): Psychiatrie ou psychanalyse. Consulté le 24 juin 2007, $<$ http://www.geocities.com/blpnow84/Selection/psypsy.html>.

Peraldi, François (1985): Corps du texte et corps érotique. Texte. 4:177-189.

PyM, Anthony (2003): Texts on translation. Henri Meschonnic. Target. 15(2):337-353. 
Quignard, Pascal (1987): La leçon de musique. Paris: Éditions Gallimard.

Saint-Germain, Christian (2007): Comprimés ou honoraires. Colloque Transfert et addiction. Centre de recherche interuniversitaire sur la littérature et la culture québécoises, Université de Montréal, 18 mai 2007.

Reık, Theodor (1975): Trente ans avec Freud. (Traduit par Evelyne Sznycer). Bruxelles: Éditions Complexe.

Seleskovitch, Danica et Lederer, Marianne (1984): Interpréter pour traduire. Paris: Didier érudition.

This, Bernard et Thèves, Pierre (1982): Comment peut-on traduire Hafiz... ou Freud? Meta. 27(1):37-59.

VenUti, Lawrence (2002): The difference that translation makes: the translator's unconscious. In: Alessandra Riccardi, dir. Translation Theories. Cambridge: Cambridge University Press, 214-241.

Wulf, Christoph (2007): Ouie. In: Michela Marzano, dir. Dictionnaire du corps, Paris: PUF, 678-681. 